\title{
A solução para o SUS não é um Brazilcare
}

\section{The solution to SUS is not a Brazilcare}

\section{La solución para el SUS no es uno Brazilcare}

Isabela Soares Santos | isantos@ensp.fiocruz.br

Fundação Oswaldo Cruz, Escola Nacional de Saúde Pública Sérgio Arouca. Rio de Janeiro, RJ. Brasil. Centro Brasileiro de Estudos de Saúde. Rio de Janeiro, RJ. Brasil.

\section{Resumo}

O Sistema Único de Saúde brasileiro implantado não recebeu todos os investimentos necessários para alcançar a magnitude prevista desde sua concepção e estabelecida na Constituição Federal de 1988. No mesmo período, o setor privado de saúde brasileiro vem recebendo cada vez mais investimentos por meio das políticas públicas do Estado. A crise econômica e os problemas pelos quais o SUS passa nos dias atuais são usados por determinados atores para justificar uma suposta necessidade de diminuir não só a pressão por financiamento, mas também a demanda de serviços públicos, e apresentar como solução a diminuição do SUS concomitante à expansão do número de pessoas com planos privados de saúde nos moldes da reforma do sistema de saúde norte-americano conhecida como Obamacare. Este artigo apresenta a falácia desse raciocínio com evidências científicas e argumentos que mostram que um maior investimento no SUS é fundamental para o desenvolvimento econômico e social do país.

Palavras-chave: políticas públicas de saúde; Sistema Único de Saúde; planos privados de saúde; mix público-privado; Obamacare; planos acessíveis; política de austeridade; gasto social; investimento público. 


\begin{abstract}
The Brazilian Sistema Único de Saúde (Unified Health System) in operation has not received all the investments needed to achieve the expected magnitude since its conception and established by Federal Constitution of 1988. In the same period, the health private sector in Brazil has received more and more investments through governmental public policies. The economic crisis and the problems faced by SUS today are used by some actors to justify a pretense necessity of reducing not only the pressure to finance but also the demand for public services, and to present as a solution to such problems a reduction of SUS concomitant with the expansion of people benefiting from private health insurance like those created with reform of the North American health care system known as Obamacare. This article shows the fallacy of reasoning in question through scientific evidences and arguments demonstrating that a greater investment in SUS is fundamental to economic and social development of Brazil.
\end{abstract}

Keywords: public policies for health care; Unified Health System; private health insurance; publicprivate mix; Obamacare; inexpensive health insurances; austerity measures; social expenditures; public investment.

\title{
Resumen
}

El Sistema Único de Salud brasileño implementado no recibió todas las inversiones necesarias para alcanzar la magnitud esperada desde su concepción y establecida en la Constitución Federal de 1988. En el mismo período, el sector privado de salud brasileño ha recibido cada vez más inversiones por el medio de las políticas públicas del Estado. La crisis económica y los problemas por los cuales el SUS ha pasado en los días actuales son utilizados por determinados actores para justificar una supuesta necesidad de reducir no sólo la presión de financiación, sino también la demanda de servicios públicos, y presentar como solución la disminución del SUS concomitantemente a la expansión del número de personas con planes privados de salud en los moldes de la reforma del sistema de salud norteamericano, conocida como Obamacare. Esto artículo presenta la falacia de ese raciocinio con evidencias científicas y argumentos que muestran que una mayor inversión en el SUS es esencial para el desarrollo económico y social del país.

Palabras clave: políticas públicas de salud; Sistema Único de Salud; planes privados de salud; mix público-privado; Obamacare; planes accesibles; política de austeridad; gasto social; inversión pública

Contribuição dos autores: A autora é responsável pelo texto.

Declaração de conflito de interesses: não há.

Fontes de financiamento: Não há

Histórico do artigo: Submetido: 15.set.2016 | Aceito: 20.set.2016 | Publicado: 30.set.2016

Licença CC BY-NC atribuição não comercial. Com essa licença é permitido acessar, baixar (download), copiar, imprimir, compartilhar, reutilizar e distribuir os artigos, desde que para uso não comercial e com a citação da fonte, conferindo os devidos créditos de autoria e menção à Reciis. Nesses casos, nenhuma permissão é necessária por parte dos autores ou dos editores. 
Nos últimos anos, o Brasil vem enfrentando uma forte crise econômica e política e, como era de se esperar, o setor de saúde sofre importantes consequências. Uma delas se passa no mercado de planos privados de saúde, com perda de quase dois milhões de clientes entre dezembro de 2014 e junho de 2016 (de 50,4 para 48,5 milhões ${ }^{1}$ ), provenientes de dois grupos populacionais. Um é formado pelos que pagavam o plano e tiveram que diminuir os gastos para sobreviver com orçamento familiar diante do aumento de custos ou, muitas vezes, por causa da redução do orçamento diante do desemprego de algum membro da família que deixa de contribuir e ainda requer apoio. O outro grupo compreende as pessoas que perderam o emprego e, com isso, o apoio financeiro do empregador para pagar o plano.

Além disso, a saúde está sempre no topo da lista das principais preocupações listadas pelas pesquisas de opinião dos últimos tempos, em todo país, tanto no âmbito nacional quanto nas campanhas municipais, como está ocorrendo neste ano, embora isso aconteça em períodos eleitorais ou não.

Em meio a essa crise, no $1^{\circ}$ semestre de 2016 o ministro da Saúde Ricardo Barros declarou à imprensa que seria preciso rever o tamanho do SUS e que não seria possível conseguir "sustentar o nível de direitos que a Constituição determina”i. Poucos meses depois, Barros apresentou como solução para sair da crise a criação de "planos acessíveis". Na audiência pública sobre o Mais Médicos ocorrida no Senado Federal em 13 de julho de 2016, Barros informou que esses seriam planos com cobertura exclusivamente ambulatorial e custariam aproximadamente $\mathrm{R} \$ 80,00$ mensais ao consumidor, e que a expectativa é de que essa medida ampliaria o mercado segurador privado em 20 milhões de segurados. Ou seja, uma forte mudança no sistema de saúde brasileiro.

Um mês depois, em outra declaração à imprensa, o ministro disse que os planos com cobertura e custo menores serviriam "para a gente colocar mais recursos no atendimento das pessoas"ii. O argumento, recorrente também entre os que defendem uma necessidade de sustentabilidade do mercado de seguros privados, é que essa medida "desafoga", "alivia” o SUS.

No mesmo mês foi publicada uma portaria do gabinete do ministro ${ }^{2}$ criando um grupo de trabalho para elaboração do projeto do plano. O grupo é composto por representantes de apenas três órgãos: Ministério da Saúde (MS), Agência Nacional de Saúde Suplementar (ANS/MS), e Confederação Nacional das Empresas e Seguros Gerais, Previdência Privada e Vida, Saúde Suplementar e Capitalização (CNSeg). Deste grupo não participam os movimentos sociais, as organizações de usuários de serviços de saúde, pacientes e profissionais de saúde, especialistas acadêmicos, tampouco o Conselho Nacional de Saúde. Podem ser convidados, mas não são membros do grupo.

Os fatos recentes expostos acima nos mostram que, ao passo que a sociedade civil é absolutamente desconsiderada na formulação da proposta e tomada de decisão de mudanças do sistema de saúde, somente são chamados para desenhar a proposta governamental os representantes do próprio governo (ANS e MS) e do mercado segurador, vinculado ao capital financeiro e que objetiva a acumulação e o lucro (CNSeg).

Além da proposta de planos acessíveis, a ANS vem estudando um projeto de diminuição dos preços mensais dos planos mediante o aumento da franquia, similar aos high deductibles propostos pela reforma do sistema de saúde dos Estados Unidos, conhecida como Obamacare e em vigor naquele país desde 2012.

Essas propostas de mudança do SUS fortalecem o setor privado de seguros com a diminuição do tamanho do SUS e a sua fragmentação e guardam clara influência das estratégias do Obamacare de expansão da clientela de seguros privados. A pergunta que se faz é se esse modelo vai melhorar nossa saúde, considerando que no caso brasileiro temos um sistema público de saúde para todos, o que não existe nos Estados Unidos. O que vamos expor nesta nota é que essa proposta faz parte de um modelo de sistema de

i Disponível em: http://www1.folha.uol.com.br/cotidiano/2016/05/1771901-tamanho-do-sus-precisa-ser-revisto-diz-novo-ministroda-saude.shtml

ii Jornal O Globo de 6/8/2016, p. 22. 
saúde e de políticas públicas que fortalece o setor privado, aumenta a fragmentação do sistema, não alivia do SUS e não serve para sociedade alguma por diversos motivos explicados a seguir.

\section{O Brasil precisa um sistema público de saúde forte e de qualidade}

Precisamos de um sistema público forte e de qualidade primeiramente porque o SUS é para ser usado por todos os brasileiros. Qualquer pessoa que precise utilizar um serviço de saúde pode ser atendida pelo SUS e não precisa pagar por ele na hora do uso. A essa condição denominamos direito universal à saúde. Trata-se de um direito de cidadania. Isso não ocorre com os planos privados de saúde, nem com os médicos, laboratórios e hospitais privados sem atendimento do SUS, pois nesses é necessário pagar para usar, seja por intermédio do plano ou pagando diretamente do próprio bolso - nesses casos, é estabelecido o direito de consumidor.

O SUS pode ter diversos problemas, mas ainda é para todos. O direito de cidadania estende-se a todas as pessoas, principalmente nas sociedades preocupadas com o bem-estar geral da população, cuja organização é determinada com base em valores de solidariedade, os quais fundamentam serviços de interesse público.

Já, no setor privado, o uso do serviço de saúde mediante o pagamento é baseado no direito de consumo e ocorre se a pessoa tiver como pagar. Do contrário, não poderá usá-lo. Nesse caso, é cada um por si, de modo a prevalecer o valor da liberdade individual traduzido como renda e riqueza. Isso acontece nos mercados privados, em que os mais ricos se beneficiam por ter maior capacidade de pagamento enquanto os mais pobres são prejudicados.

Na prática, todos os sistemas de saúde do mundo misturam o público e o privado. É importante compreender, em cada sistema, se aquilo que é de interesse de todos (interesse público) consegue prevalecer sobre o interesse de alguns (interesse privado).

No Brasil, por mais problemas que apresente e por mais imbricado que esteja com o setor privado, o SUS ainda é um sistema de saúde de interesse público para todos de brasileiros. Nenhum outro país desse tamanho tem sistema público para todos. Portanto, o SUS é nosso e precisa ser melhorado.

O setor privado de planos e seguros de saúde e de prestação de serviços não é um sistema, não funciona como tal e nem tem esse objetivo, mas sim o lucro de cada empresa, sendo que as empresas são concorrentes entre si, o que configura um setor fragmentado.

O SUS é um sistema. E está organizado como tal, em rede, com ações, serviços, clínicas e postos de saúde, hospitais, centros de serviços de apoio à diagnose e terapia (SADT), profissionais que executam as ações e os serviços, recursos físicos, e políticas orientadoras articuladas. Sozinhos, esses pontos não formam uma rede, somente quando seus pontos são interligados (com sistema de informação, logística, comunicação etc.) é que constituem os fios da rede. Isso não ocorre no setor privado pela sua natureza fragmentada ${ }^{3}$.

Além disso, a lógica da atenção à saúde não se resolve se estiver centrada nos eventos hospitalares e agudos, como costuma ocorrer na assistência prestada pelo setor privado, que é orientado pela lógica de pagamento por serviço executado. É preciso orientar a atenção pela lógica da Atenção Primária à Saúde (APS). A maior parte dos problemas de saúde pode ser resolvida na APS. Além disso, um estudo de Barbara Starfield ${ }^{4}$, que é referência neste tema, comparou 11 países e mostrou que os sistemas de saúde orientados pela APS estão associados a uma maior satisfação da população, um menor uso de medicamentos e melhores níveis de saúde.

Também precisamos de um sistema público para viabilizar a organização e a gestão do sistema. Sem um sistema seria inviável organizar a atenção à saúde do SUS em mais de cinco mil municípios, mais de 44 mil postos e centros de saúde, cerca de 41 mil policlínicas e clínicas especializadas, 20 mil estabelecimentos de SADT, além dos mais de seis mil hospitais e mil prontos-socorros para mais de 200 milhões de brasileiros. 
Outro motivo é ter escala econômica. Alguns indivíduos necessitam gastos com saúde muito maiores que outros. Diluir esses gastos gera viabilidade econômica, que vai ser tão maior quanto maior a população. Essa lógica é reforçada dentro de um contexto em que os custos com saúde são cada vez maiores em razão do desenvolvimento tecnológico e devido a diversas mudanças que reforçam a necessidade da diluição dos gastos com saúde. Entre essas mudanças, destacam-se o envelhecimento populacional, o aumento das doenças crônicas, a aceleração da urbanização não planejada, a gentrificação, o crescimento dos modos de vida pouco saudáveis.

Um sistema público de saúde éfundamental também para serem estabelecidas regras mínimas de segurança e qualidade dos serviços realizados e, ainda, dos recursos físicos e humanos que oferecem os serviços. O estabelecimento dessa qualidade não pode ser previsto por cada empresa de saúde, seja um estabelecimento ou empresa de plano privado; tem de ser realizado por entidade que represente o interesse coletivo e público, e não o interesse privado do lucro. Então, só é possível ser realizado por intermédio do Estado.

Além disso, também é importante por questões relacionadas ao desenvolvimento econômico do país que o sistema público de saúde seja bom, pois interessa a todos que ele garanta uma população saudável e com qualidade de vida. Alguns países já atentam para a necessidade de prover sustentabilidade às políticas sociais, de modo a garantir o acesso da população aos serviços de educação, saúde, previdência, saneamento e assistência social, para que as suas sociedades possam se desenvolver inclusive economicamente e possam enfrentar a pobreza, gerar empregos e produzir riquezas. Veremos mais sobre esse ponto na próxima seção.

\section{Evidência científica sobre resultados da política econômica de austeridade}

Dois meses atrás, o ministro da Fazenda Henrique Meirelles disse que "As despesas com educação e saúde são itens que, na prática, junto com a previdência, inviabilizaram um controle maior das despesas nas últimas décadas”iii. Disse também, na mesma entrevista, que "educação e saúde inviabilizam ajustes". Essas declarações fazem parte da argumentação da proposta de que, para sair da crise, é preciso cortar os gastos sociais, manter os juros altos, ter meta de inflação, superávit primário, fazer privatizações, abrir o mercado para os grupos financeiros internacionais (capital estrangeiro). Essa é a proposta de equilíbrio das contas do governo, chamada de política de austeridade ou política ortodoxa, e conhecida como política de austeridade da agenda neoliberal que está sendo aplicada na Grécia e na Espanha como alternativa de enfrentamento da crise econômica em que esses países se encontram desde 2008.

O que os defensores da política da austeridade não sabem ou não nos dizem é que, se cortarmos os gastos sociais - e saúde é um importante gasto social -, vai acontecer um desastre com aqueles indicadores que levamos anos para melhorar no país.

Recentemente foram desenvolvidos estudos científicos que mostram a importância das políticas sociais, tanto pelos valores humanitários que as caracterizam, como para a sua própria sustentabilidade e para o desenvolvimento econômico dos países.

O estudo de Reeves e outros ${ }^{5}$ sobre a política econômica em 27 países da União Europeia, no período de 1995 a 2011, realizou uma análise multivariada dos determinantes políticos, econômicos e do sistema de saúde sobre as mudanças nos gastos com saúde ocorridas naqueles países. A análise gerou o indicador "multiplicador fiscal", que mostra o montante de recursos que se obtém de volta com diferentes gastos públicos. O resultado mostrou que os melhores índices multiplicadores vêm das despesas com educação e saúde, ao passo que os piores provêm dos gastos com defesa.

O que ocorre com a Grécia mostra bem a tragédia da política de austeridade e é também um caso exemplar no estudo de Stuckler e Basu' ${ }^{6}$ que analisaram as restrições nos gastos com saúde, no período

iii Entrevista concedida dia 01/07/2016 à Rádio Estadão. 
de 2009 a 2012, com tratamentos, diagnósticos, medicamentos, prevenção e saúde materno-infantil. Verificaram grandes aumentos nas taxas de mortalidade infantil, de suicídio, colapso na prevenção contra o HIV que estaria transformando a Grécia num epicentro de disseminação do vírus tamanho foi o aumento das infecções, além do aumento da população sem teto (homeless). Já no caso da Islândia, que aumentou os gastos com políticas de proteção social e com o suporte de proteção aos pobres, verificaram que não houve perda no acesso aos serviços de saúde, e se obteve aumento das horas de sono, diminuição da ingestão de fast food com aumento do consumo de peixe, e ainda a entrada do país para o World Happines Report ${ }^{7}$ pelos indicadores de felicidade, que guardam grande relação com a proteção social.

Os resultados de Stuckler e Basu ${ }^{6}$ e Reeves e outros ${ }^{5}$ mostram como os cortes nos orçamentos de saúde em tempos de crise econômica podem piorar a condição de vidas dos países, provocando uma "tragédia humana".

No caso dos países da America Latina, funcionários do Fundo Monetário Internacional (FMI) defendem que as políticas neoliberais aumentam a desigualdade, prejudicam a sustentabilidade do crescimento e ainda colocam em risco a expansão duradoura da economia desses países: "a experiência do Chile e de

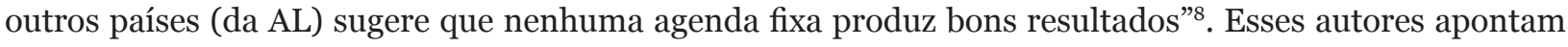
que a política do FMI não teria sido exitosa no combate à pobreza e à desigualdade.

Os estudos mencionados mostram que, quando se faz cortes na saúde em momento de crise econômica, há um aumento do número de mortes, dos surtos de infecções por tuberculose e por HIV, e das doenças infectocontagiosas em geral. Também aumenta o risco de retorno de doenças que já estavam erradicadas, aumentam os índices de alcoolismo e de suicídio, os problemas de saúde mental, ou seja, uma tragédia para uma população sofrida de um país em crise. Além disso, tais estudos mostram que os cortes nos gastos sociais aumentam a desigualdade no país e colocam em risco a possibilidade de uma expansão duradoura assim como prejudicam a sustentabilidade do crescimento.

Além de mostrarem que a saúde não deve ser cortada em situação de crise, esses estudos mostram que investir em saúde pode ser considerada uma oportunidade de gerar economia com desenvolvimento de tecnologias, emprego e maior qualidade de vida, que são fundamentais para enfrentar crise. Os autores sugerem que manter ou até mesmo aumentar o gasto com saúde contribui para o país voltar crescer mais rapidamente e de forma mais duradoura. Nesse sentido, é possível afirmar que nos momentos de crise os governos devem investir mais em saúde, porque é quando as pessoas mais precisam e porque a saúde contribui para um desenvolvimento mais duradouro da economia do país.

É importante compreender que essa política de austeridade ganha grande força como resposta à crise econômica mundial e coincide com o esforço da Organização Mundial de Saúde a partir de 2005 para emplacar a proposta de Universal Health Coverage - UHC (Cobertura Universal de Saúde - CUS) para todos países do mundo. A CUS baseia-se na expansão do mercado privado de saúde sob os argumentos de que "grande proporção da população está disposta a pagar por serviços de saúde do setor privado" e "fortes agentes do mercado estão dispostos a pressionar para que haja aumento de financiamento público e privado, especialmente como os países de média e baixa renda adotam políticas para financiar o seguro de saúde como um meio para a CUS" (tradução livre) ${ }^{9}$. Ou seja, o efeito da crise econômica no mercado segurador internacional leva à busca de novas clientelas e nesse sentido as maiores economias fora do hemisfério Norte são o alvo mais adequado dessa política, colocando neste mapa países Brasil, Índia e África do Sul. 


\section{A saúde do setor privado de saúde do brasil depende do setor público brasileiro}

O SUS real que temos não é o SUS constitucional. Desde a aprovação da máxima "Saúde como direito de todos e dever do Estado", na Constituição Federal de 1988, e também que "A saúde é livre à iniciativa privada", o SUS tem de competir com o mercado privado de saúde, porém em situação de desvantagem, como bem mostra o documento do Centro Brasileiro de Estudos de Saúde ${ }^{10}$ :

- A maior parte do gasto com saúde no Brasil é no setor privado; enquanto entre as 10 maiores economias do mundo, só na Índia o gasto privado em saúde é maior que no Brasil;

- O orçamento do MS é menor que o faturamento das empresas de planos privados de saúde. O MS, porém, destina-se a todos os brasileiros, e os planos a um quarto da população;

- Em 11 anos, a proporção do financiamento federal relativa ao financiamento total do SUS caiu de $58,4 \%$ para apenas $45,4 \%$;

- Aisenção do imposto de renda para os gastos com saúde privada é enorme (equivalente a 22,5\% de todo o gasto federal com saúde em 2011). Naquele ano, metade da isenção era relativa às despesas com planos de saúde;

- Os gastos do governo federal com assistência privada à saúde para os servidores públicos e familiares equivalem a $5 \%$ do valor total do orçamento do MS;

- As empresas de planos privados de saúde têm forte poder econômico e financeiro e gastam bilhões de reais em publicidade;

- Os principais fornecedores de equipamentos, medicamentos e insumos biomédicos são privados;

- O Brasil é o quarto maior mercado de produtos farmacêuticos de todo o mundo, e o SUS é comprador de uma grande fatia desse mercado;

- Os prestadores privados de serviços de saúde são responsáveis por grande parte dos leitos de internação hospitalar do SUS, e ainda há vários programas públicos de apoio financeiro a essas entidades;

- O66\% dos equipamentos de diagnóstico e terapia encontram-se sob controle do setor privado, e 62\% dos leitos hospitalares existentes no Brasil eram privados em 2013;

- Os gestores privados de saúde vêm sendo fortalecidos pela contratação de Organização da Sociedade Civil de Interesse Público (Oscip) e Organizações Sociais (OS), sendo crescente sua atuação na gestão de hospitais, centros de saúde, Unidades de Pronto Atendimento (UPA) e unidades de saúde da família, complexificando a privatização do SUS "por dentro", no que ainda era público, a saber, a gestão.

Além disso, os planos privados são um estelionato. Grande parte das pessoas que têm plano, o têm por intermédio do emprego, mesmo que paguem parte da mensalidade ou toda ela. Quando se aposentarem, seus orçamentos diminuirão, mas aumentarão seu custo de vida, sua necessidade de serviços de saúde e seus gastos com saúde. Provavelmente não conseguirão continuar pagando por esses planos. A única saída para nós e nossos filhos, assim como para todos os brasileiros, é um SUS melhor.

Esses são exemplos de importantes resultados de ações concorrenciais que o SUS tem com o setor privado, em situação desfavorável. São situações como essas que precisamos enfrentar. Por exemplo, só o fato de as pessoas terem desconto no imposto de renda para pagar assistência privada à saúde já é um forte motivador para que justamente a população mais formadora de opinião tenha plano e deixe de lutar pelo SUS. Que futuro espera essas pessoas? 


\section{Ataques recentes ao sus que favorecem o setor privado}

além da concorrência desleal que enfrenta com o setor privado, desde sua criação o SUS vem sofrendo uma infinidade de ataques que claramente visam ao seu desmonte. Destacam-se o subfinanciamento com a Desvinculação das Receitas da União (DRU) em 20\%, inicialmente chamado de Fundo Social de Emergência (1994), depois denominado de Fundo de Estabilidade Fiscal e, desde 2003, DRU. Atualmente, a Proposta de Emenda Constitucional (PEC) 143, de 2015, estabelece a desvinculação num percentual maior, ou seja, de 30\% da receita para perdurar até o ano de 2023. A Emenda Constitucional $\mathrm{N}^{\circ} 29$, de 2000, estabeleceu o percentual mínimo do orçamento dos estados e municípios a ser gasto em saúde, mas não alcançou os 10\% pleiteados para a União, nem mesmo com mais de dois milhões de assinaturas em Projeto de Lei de iniciativa popular apresentado pelo Movimento Saúde +10 .

É grave a promiscuidade entre interesses privados e públicos, uma vez que as empresas de planos privados de saúde são importantes financiadoras de campanhas eleitorais. Nesse sentido, em 2014, o então deputado Eduardo Cunha foi autor de emenda à Medida Provisória (MP) No 627 de 2013 para anistiar os planos privados, posteriormente vetada pela ex-presidenta Dilma Rousseff. O mesmo deputado apresentou a PEC 451/2014, ainda em tramitação no Congresso Nacional, que obriga o empregador a oferecer plano privado aos trabalhadores com quem mantém vínculo empregatício em todo o país. No início de 2015, esse mesmo deputado negou o pedido de abertura de CPI dos planos privados, e foi aprovada pelo Congresso a Lei $N^{0} 13.097$ que permite a participação direta ou indireta e controle do capital estrangeiro na saúde. Notese que essa lei é fruto da MP No 656 de 2014, que teve emenda apresentada por um deputado do PMDB da Paraíba com campanha eleitoral financiada pelo Bradesco.

Em agosto de 2015, foi apresentado o documento “Agenda Brasil”, fruto do acordo que o PMDB estava promovendo e que desembocou no golpe parlamentar ocorrido em 2016. Nesse documento, entre outras propostas anticonstitucionais estava a de cobrança diferenciada de procedimentos do SUS segundo a faixa de renda do usuário.

Mais recentemente, as bases da seguridade social estabelecidas na Constituição foram colocadas em xeque com a PEC 241/2016, que estabelece, para os próximos 20 anos, teto para as despesas primárias do governo, entre as quais estão contempladas as que se referem à saúde, educação etc. Um dos pontos mais graves dessa proposta se refere ao tempo durante o qual perdura e em qual modelo de sociedade implica, como afirma David" ${ }^{11}$ : "duração de 20 anos da proposta, explicitando que não se trata de uma ação para lidar com uma crise econômica, mas uma atitude deliberada para mudar o Estado de Bem-Estar Social, preconizado na Constituição Federal, para um Estado Mínimo, que não garante direitos humanos, sociais, econômicos, culturais e ambientais".

\section{Evidência científica em arranjos público-privados de saúde com sistemas públicos universais e planos privados de saúde}

Há estudos internacionais ${ }^{12-16}$ que evidenciam que a convivência de cobertura para seguro privado com um sistema público, chamada cobertura duplicada, gera alguns efeitos, tais como: (i) o sistema público fica com maiores e mais complexas filas de espera; (ii) o arranjo público-privado contribui para a iniquidade na oferta, no acesso e no uso dos serviços; (iii) incentiva o desenvolvimento do setor privado nos serviços em que a população tem dificuldade de acesso no sistema público, geralmente consultas a especialistas, cirurgias eletivas, SADT e medicamentos; (iv) não diminui a pressão da demanda por serviços do sistema público e, tampouco, pelo financiamento desse sistema; (v) não contribui para a preservação dos objetivos gerais do sistema de saúde - de universalidade, integralidade e equidade; de colaboração positiva para os

iv Disponível em: http://www12.senado.leg.br/noticias/materias/2015/08/10/a-agenda-brasil-sugerida-por-renan-calheiros 
resultados de saúde, o desenvolvimento do próprio sistema de saúde e objetivos sociais, como a melhoria das condições de vida da população. Ao contrário, corrói esses objetivos do sistema. Portanto, não há evidência de que o seguro privado alivie os sistemas públicos universais.

\section{Considerações finais}

Nos últimos anos o Brasil melhorou em muita coisa. No caso da saúde, mesmo com todos os problemas do SUS, o fato de termos um sistema público de saúde nos levou a melhorar indicadores importantes de saúde. Sim, queremos mais. Mas não podemos deixar de ver as reduções na taxa de mortalidade infantil, na taxa de desnutrição em crianças e na ocorrência das doenças infectocontagiosas, parasitárias e as imunopreveníveis ${ }^{17-19}$.

Também melhoramos com o aumento da expectativa de vida e, portanto, de pessoas idosas. Ao mesmo tempo, tivemos aumento da população em idade ativa e diminuição da fecundidade, o que gera a mudança na distribuição demográfica da população, a transição demográfica. Tudo isso demanda diferentes serviços de saúde e em maior quantidade, mas é um problema muito bem-vindo, porque é fruto de uma sociedade que vive mais e com melhores condições de vida se comparada à das gerações anteriores.

O Brasil também melhorou com a diminuição das desigualdades. Nosso país deixou de estar no mapa mundial da fome. Embora a pobreza ainda seja grande, diminuiu a quantidade de pessoas que viviam na miséria. Aumentamos a escolaridade e diminuímos o analfabetismo.

A que se devem essas melhorias? A diversos fatores, mas o principal foi a Constituição Federal de 1988 que trouxe os direitos sociais para todos os cidadãos, independentemente de raça, renda ou gênero, entre eles o direito à saúde para toda a população.

Como vimos, na relação público-privada no setor de saúde brasileiro, o setor privado sobrevive graças ao investimento público que nele é feito e, ao mesmo tempo, em detrimento do investimento no SUS. O problema disso é que, sem um sistema público de alta qualidade, o país investe menos nas condições de saúde da população, gerando não só os problemas reais de saúde para as pessoas, mas também maiores gastos nessa área com problemas que poderiam ser evitados e, ainda, uma população menos saudável. Esse tipo de política de baixo investimento em políticas que são para todos "é um tiro no pé”, afeta inclusive a capacidade de desenvolvimento econômico do país, tanto a atual como a futura.

Propostas como a de reduzir o tamanho do SUS ou ampliar o número de pessoas com planos privados não vão aliviar ou melhorar o SUS. O SUS não deve funcionar como sistema complementar ao setor privado. Ao contrário, o SUS é um sistema imenso e extremamente complexo, um dos maiores sistemas de saúde pública universal do mundo, que atende com uma gama de serviços cada vez mais ampla a uma população enorme num território extenso e diferente como o do Brasil.

Considerando as décadas anteriores ao SUS e depois de sua criação, o sistema público de saúde brasileiro só cresceu e se aprimorou, apesar dos fortes embates necessários para persistir e sobreviver às políticas advindas de todos últimos governos federais, que resultaram no desfinanciamento federal do SUS, no investimento no trabalho terceirizado e desinvestimento no trabalho público, que afeta fortemente as condições de trabalho do quadro de servidores do SUS, entre inúmeras outras políticas contrárias ao fortalecimento do SUS como direito de cidadania.

Enfim, se quisermos um país forte e de forma duradoura, é preciso grandes investimentos na área social com efeitos no universo da população. Para isso é preciso da ação do Estado no sentido de tornar realidade os direitos sociais. No caso do SUS, tornar real o SUS constitucional. Certamente a grande maioria da população brasileira deseja saúde pública e de qualidade para todos os brasileiros e isso só é possível com um sistema público universal forte e de qualidade para todos. 


\section{Referências}

1. IBGE. Sistema de Informações de Beneficiários, SIB/ANS/MS. [citado jun 2016] Disponível em http://ces.ibge.gov.br/base-de-dados/metadados/ministerio-da-saude/sistema-de-informacoes-debeneficiarios-sib.html

2. Brasil. Ministério da Saúde, Gabinete do Ministro. Portaria n. ${ }^{\circ} 1482$ de 4 de agosto de 2016. Institui Grupo de Trabalho para discutir projeto de Plano de Saúde Acessível. Diário Oficial da União, Brasília (DF), 05 ago. 2016, Seção 1:26.

3. Rodrigues PHA, Santos, IS. Saúde e cidadania: uma visão histórica e comparada do SUS. Rio de Janeiro: Atheneu; 2011.

4. $\quad$ Starfield B. Is primary care essential? Lancet. 1994 Oct 22;344(8930):1129-33.

5. Reeves A, McKee M, Basu S, Stuckler D. The political economyof austerity and healthcare: Cross-national analysis of expenditurechanges in 27 European nations 1995-2011. Health Policy. 2014 Mar;115(1):1-8.

6. Stuckler D, Basu S. A economia desumana: porque mata a austeridade. Bizâncio, Portugal; 2014.

7. World Happiness Report. 2016. [citado 15 set. 2016] Disponível em: http://worldhappiness.report/.

8. Ostry JD, Longani P, Funceri D. Neoliberaism: Oversold? Finance \& Development. 2016 Jun, 53(2):38-41.

9. Rockefeller Foundation. Future Health Markets: a meeting statement from Bellagio. 2012. [citado 15 set. 2016] Disponível em: https://static.squarespace.com/static/5034f626e4b09af678ebcd47/52c594f5e4b01a0 9cde0f896/52c594f5e4b01a09cde0faf7/1355906022603/bellagio-future-health-markets-statement-final.pdf

10. Manifesto do Centro Brasileiro de Estudos de Saúde em defesa do direito universal à saúde - saúde é direito e não negócio. Versão 1.2. 2014. [citado 15 set. 2016] Disponível em: http://cebes.org.br/ site/wp-content/uploads/2014/09/Manifesto Cebes Sa\%C3\%BAde \%C3\%A9 direito e n\%C3\%A30 neg\%C3\%B3cio.pdf

11. David, G. A austeridade atinge os direitos humanos na América Latina. Carta Capital. 5 set 2016. [citado 15 set. 2016] Disponível em: http://politike.cartacapital.com.br/a-austeridade-atinge-os-direitoshumanos-na-america-latina/

12. Organisation for Economic Co-operation and Development (OECD). Private Health Insurance in OECD Countries. Paris: OECD; 2004.

13. Mossialos E, Thompson SMS. Voluntary health insurance in the Europe Union. Copenhagen: World Health Organization (WHO) Regional Office for Europe on behalf of the European Observatory on Health Systems and Policies; 2004.

14. Couffinhal A. Concurrence en assurance santé: entre efficacité et sélection (Competition in health insurance: between efficiency and selection). [Dissertation]. Paris, Université Paris IX-Dauphine; 1999

15. Colombo F, Tapay N. Private Health Insurance in OECD countries: the benefits and costs for individuals and health systems. OECD Health Working Papers n. 15. Paris: OECD; 2004.

16. Tuohy $\mathrm{CH}$, Flood $\mathrm{CM}$, Stabile M. How does private finance affect public health care systems? Marshaling the evidence from OECD Nations. Journal of Health Politics, Policy and Law, 2004; 29(3): 359-396.

17. Fundação Oswaldo Cruz. A saúde no Brasil em 2030: prospecção estratégica do sistema de saúde brasileiro: estrutura do financiamento e do gasto setorial. Rio de Janeiro: Fiocruz: Ipea: Ministério da Saúde: Secretaria de Assuntos Estratégicos da Presidência da República; 2013. v2. Capítulo II: Cenário Sociodemográfico em 2022/2030 e Distribuição Territorial da População. Uso e Ocupação do Solo.p.85-90.

18. Fundação Oswaldo Cruz. A saúde no Brasil em 2030: prospecção estratégica do sistema de saúde brasileiro: estrutura do financiamento e do gasto setorial. Rio de Janeiro: Fiocruz: Ipea: Ministério da Saúde: Secretaria de Assuntos Estratégicos da Presidência da República; 2013. v2. Doenças Transmissíveis, Endemias, Epidemias e Pandemias. p.97-107.

19. Fundação Oswaldo Cruz. A saúde no Brasil em 2030: prospecção estratégica do sistema de saúde brasileiro: estrutura do financiamento e do gasto setorial. Rio de Janeiro: Fiocruz: Ipea: Ministério da Saúde: Secretaria de Assuntos Estratégicos da Presidência da República; 2013. v2. Esboços para um Cenário das Condições de Saúde da População Brasileira 2022/2030. p. 249-306. 